\title{
Expression, Localization, and Phosphorylation of Akt1 in Benign and Malignant Thyroid Lesions
}

\author{
Anna Krześlak • Lech Pomorski • Anna Lipińska
}

Published online: 6 September 2011

(C) The Author(s) 2011. This article is published with open access at Springerlink.com

\begin{abstract}
The serine/threonine protein kinase Akt is a key molecule in the phosphatidyl inositol 3-kinase pathway that is often overactivated in human cancers. Three Akt isoforms (Akt1, Akt2, Akt3) have been identified in human cells and they show different distribution and have nonredundant functions. The aim of this study was to determine whether the expression, phosphorylation, and localization of Akt1 isoform in human thyroid malignant lesions are different from those in benign lesions. Nuclear and cytoplasmic fractions were isolated from tissue samples and Western blot method was used to detect Aktl presence in both cellular fractions. Akt1 expression was also assessed by ELISA method. To estimate Akt1 phosphorylation, kinase was immunoprecipitated from cell lysates and tested with anti-phospho-Akt antibodies. The Akt1 expression in majority of thyroid cancer samples was significantly higher than in benign lesions $(p<0.05)$. Akt1 both in differentiated cancers (follicular and papillary) and benign lesions was localized mainly in cytoplasmic fraction. In two of three anaplastic cancer samples Akt1 was predominantly localized in nucleus. The ratio of phosphorylated Akt1 to total Akt1 was lower in cancers than in non-neoplastic lesions and adenomas. Thus, although Akt1 seems to be overexpressed in thyroid neoplasms, its high phosphorylation is not characteristic for thyroid cancers.
\end{abstract}

\footnotetext{
A. Krześlak $(\bowtie) \cdot$ A. Lipińska

Department of Cytobiochemistry, University of Lodz, Pomorska 141/143,

90-236 Lodz, Poland

e-mail: krzeslaka@interia.pl

L. Pomorski

Department of General and Oncological Surgery,

Medical University of Lodz,

Parzeczewska 35,

95-100 Zgierz, Poland
}

Keywords Thyroid cancer Akt1 expression . Phosphorylation · Localization

\section{Introduction}

Follicular epithelial cell-derived thyroid tumors are the most common endocrine neoplasms and can be classified into thyroid adenoma (ADE), papillary thyroid cancer (PTC), follicular thyroid cancer (FTC), and anaplastic thyroid cancer (ATC). The papillary and follicular thyroid carcinomas constitute the majority of thyroid carcinomas and belong to well-differentiated thyroid carcinomas. ATC is undifferentiated and is the most aggressive thyroid cancer that may arise de novo or from one of the differentiated cancers [1]. The pathogenesis and progression of thyroid neoplasms are characterized by the alteration of multiple signaling pathways and by abnormalities in a variety of tumor suppressor genes and cell cycle proteins [2]. Increased activity of phosphatidyl inositol 3-kinase (PI3K)/Akt pathway was frequently observed in thyroid cancers [3]. The activation of the PI3K/Akt protein kinase pathway appears to play an important role both in the development of thyroid tumors and in their progression [3]. It is suggested that oncogenic activation of the PI3K/Akt pathway drives transformation of adenoma to FTC and then to ATC. Moreover, PTC develops de novo after activation of MAP kinase pathway by oncogenic genetic alterations, such as $B R A F$ mutation, and its transformation to ATC may be facilitated by PI3K/AKT pathway [1].

Akt is a serine/threonine protein kinase activated by a variety of growth factors, including insulin, insulin-like growth factor-I, and epidermal growth factor, via the phosphatidylinositol 3-kinase pathway and plays a role in tumorigenesis by inhibiting apoptosis and mediating cell 
proliferation [2]. Full activity of Akt is achieved by phosphorylation at Thr308 and Ser473. Phospho-Akt (pAkt) protects cells from apoptosis by inactivation of apoptotic cascade components, such as proapoptotic Bad, caspase-9, and members of the forkhead transcription factor family. Furthermore, Akt has been implicated in regulating metastasis, which is an important process in cancer development [4, 5].

Three Akt isoforms (Akt1, Akt2, and Akt3) have been identified in mammalian cells and each is transcribed from separate genes. The Akt isoforms show significant homology to each other but they have different distribution. In physiological conditions, Akt1 and Akt2 seem to be ubiquitously expressed, whereas Akt3 has more restricted distribution, with predominance toward the heart, kidney, brain, testes, lung, and skeletal muscle [6]. Murine gene deletion models demonstrate that Akt isoforms have nonredundant functions. Whereas Akt1 null mice have growth retardation, mice lacking Akt2 show abnormal glucose homeostasis and diabetic phenotype and mice lacking Akt3 have reduced brain size [7-9].

In the normal thyroid, all three Akt isoforms are expressed but Akt1 is the predominant isoform at both mRNA and protein levels [2]. Akt1 seems to be also a principal overexpressed and overactivated isoform of Akt in thyroid cancers compared to normal tissue. It is suggested that activation of this isoform is associated with tumor invasion and metastasis in follicular and papillary cancers [10, 11].

The aim of this study was to determine whether the expression, phosphorylation, and localization of Akt1 in human thyroid malignant lesions are different from those in benign tumors and non-neoplastic tissues.

\section{Materials and Methods}

\section{Surgical Specimens}

Surgical speciments were obtained from 45 patients (10 males and 35 females), who underwent surgery for nodular thyroid disease. Thyroid specimens from patients were rapidly frozen and stored at $-80^{\circ} \mathrm{C}$. All tissues were carefully check and evaluated by an experienced pathologists. The studies were preformed on 16 specimens of nonneoplastic lesions (nodular goiters), 6 cases of follicular adenomas, 4 follicular, 16 papillary, and 3 anaplastic carcinomas.

\section{Isolation of Cytoplasmic and Nuclear Fractions}

Thawed tissue samples were homogenized for $3 \mathrm{~min}$ in Potter's homogenizer in 10 volumes of $0.25 \mathrm{M}$ sucrose containing $5 \mathrm{mM} \mathrm{MgCl}, 0.8 \mathrm{mM} \mathrm{KH}_{2} \mathrm{PO}_{4}$ (pH 6.8), $0.5 \%$
Triton X-100, and protease inhibitors. The homogenates were centrifuged at $800 \times g$ for $7 \mathrm{~min}$. The crude nuclear pellet was suspended in 10 volumes of $2.2 \mathrm{M}$ sucrose, $5 \mathrm{mM} \mathrm{MgCl}_{2}$ and centrifuged at $40,000 \times \mathrm{g}$ for $45 \mathrm{~min}$. The purity and integrity of the nuclei were checked by phasecontrast light microscopy. The supernatant obtained after separation of nuclei from homogenate of thyroid pathological specimens was centrifuged at $2,500 \times \mathrm{g}$ for $10 \mathrm{~min}$ to pellet any remaining nuclei. The supernatant obtained after this centrifugation was considered as cytoplasmic fraction. The cytoplasmic and nuclear proteins samples were mixed with solubilizing buffer and heated in a boiling water bath for $5 \mathrm{~min}$ and run on SDS-PAGE.

\section{Enzyme Linked Immunosorbent Assay}

For semi-quantitative analysis of Akt1 expression in cytoplasmic fractions, enzyme linked immunosorbent assay (ELISA) method was used. Samples of cytoplasmic fraction to be assayed for the presence of Aktl were diluted to a final concentration of $2.5 \mu \mathrm{g} / \mathrm{ml}$ in $0.1 \mathrm{M}$ carbonate buffer, $\mathrm{pH}$ 9.8. The diluted samples $(100 \mu \mathrm{l})$ were added to the wells of the 96-well microtiter polystyrene plates (Bio-Rad, Hercules, USA). The attachment of proteins to the wells was effected by overnight incubation at $4^{\circ} \mathrm{C}$ and then the plates were washed three times with TBS buffer (Trisbuffered saline) containing $0.05 \%$ Tween 20 (TTBS). The nonspecific binding sites were blocked by the addition of $200 \mu \mathrm{l}$ of $2 \%$ BSA in TTBS buffer to each well, followed by incubation for $1 \mathrm{~h}$ at room temperature. Microtiter plates were again washed three times with TTBS and incubated during $2 \mathrm{~h}$ at room temperature with $100 \mu \mathrm{l}$ per well of the mouse monoclonal anti-Aktl antibody (Santa Cruz Biotechnology, Santa Cruz, CA, USA) diluted in TTBS 1:1,000. After washing the plates three times with TTBS buffer, the goat anti-mouse antibodies conjugated with horseradish peroxidase $(100 \mu \mathrm{l}$ per well) (Santa Cruz Biotechnology) were added (dilution of antibodies 1:5,000). The plates were incubated for $1 \mathrm{~h}$ at room temperature and washed with three changes of TTBS. The color reaction was developed by adding $100 \mu \mathrm{l}$ per well of peroxidase substrate $[0.04 \%$ 1, 2-phenylenediaminedihydrochloride/OPD/(Sigma-Aldrich Co, St Louis, MO, USA) in $0.1 \mathrm{M}$ citric acid phosphate buffer ( $\mathrm{pH}$ 5.0) and $\mathrm{H}_{2} \mathrm{O}_{2}$ ]. The reaction was terminated by addition of $150 \mu \mathrm{l}$ $\mathrm{H}_{2} \mathrm{SO}_{4}$ to each well. The absorbance of the wells was measured at $490 \mathrm{~nm}$ with an automatic microplate reader (Bio-Rad, model 550).

Immunoprecipitation

Thyroid specimens (200 mg) were homogenized in lysis buffer (1\% SDS, 5 mM EDTA, 1\% 2-mercaptoethanol, 
$1 \mathrm{mM}$ PMSF, $1 \mathrm{mg} / \mathrm{ml}$ aprotinin) for 3-5 $\mathrm{min}$. Lysate samples $(700 \mu \mathrm{g})$ were incubated $2 \mathrm{~h}$ at $4^{\circ} \mathrm{C}$ under agitation with anti Akt1 monoclonal antibody, and then, $20 \mu \mathrm{l}$ of Protein G Plus Agarose reagent (Santa Cruz Biotechnology) was added to each sample. The lysate-beads mixtures were incubated overnight at $4^{\circ} \mathrm{C}$. After centrifugation, the supernatant was removed, the beads were washed three times with TBS, and $30 \mu$ l of loading buffer was added. The samples were boiled for $5 \mathrm{~min}$ and run on SDS-PAGE.

\section{Western Blot}

The samples $(50 \mu \mathrm{g}$ protein per lane) of cytoplasmic and nuclear proteins of different types of thyroid lesions or Akt1 immunoprecipitates were resolved by $8 \%$ SDS-PAGE and electroblotted onto Immobilon-P transfer membranes (Millipore, Bedford, Massachusetts, USA). The blots were incubated with the mouse monoclonal anti-Akt1 antibody or rabbit polyclonal anti-phospho-Akt (Ser473) antibodies in a 1:1,000 dilution. After being washed three times with TBST, the membranes were incubated $1 \mathrm{~h}$ with goat antimouse or anti-rabbit antibodies conjugated with horseradish peroxidase which were added in a 1:5,000 dilution. The membranes were again washed three times with TBST. Proteins were visualized on X-ray film by enhanced chemiluminescence method. Gel-Pro ${ }^{\circledR}$ Analyzer software (Media Cybernetics Inc., USA) was used for densitometry analysis of protein bands. Intensity of protein bands quantified by densitometry was used to estimate the ratio of phospho-Akt1 to total Akt1.

\section{Statistical Analysis}

Where appropriate, experimental data are expressed as mean \pm SD. Statistical analysis was performed using the computer program STATISTICA version 9.0 (StatSoft, Poland). The groups were compared using Mann-Whitney rank sum test. $P$ value $<0.05$ was considered statistically significant.

\section{Results}

The results of cytoplasmic Akt1 expression in thyroid lesions obtained with the ELISA method are shown in Fig. 1. Akt1 expression level was very variable in each group of thyroid, i.e., non-neoplastic lesions (nodular goiters; NN), follicular adenomas (ADE), papillary carcinomas (PTC), follicular carcinomas (FTC), and anaplastic carcinomas (ATC) (Fig. 1a). However, Akt1 expression in majority of papillary thyroid cancer samples was significantly higher than in non-neoplastic lesions $(p<0.05)$.
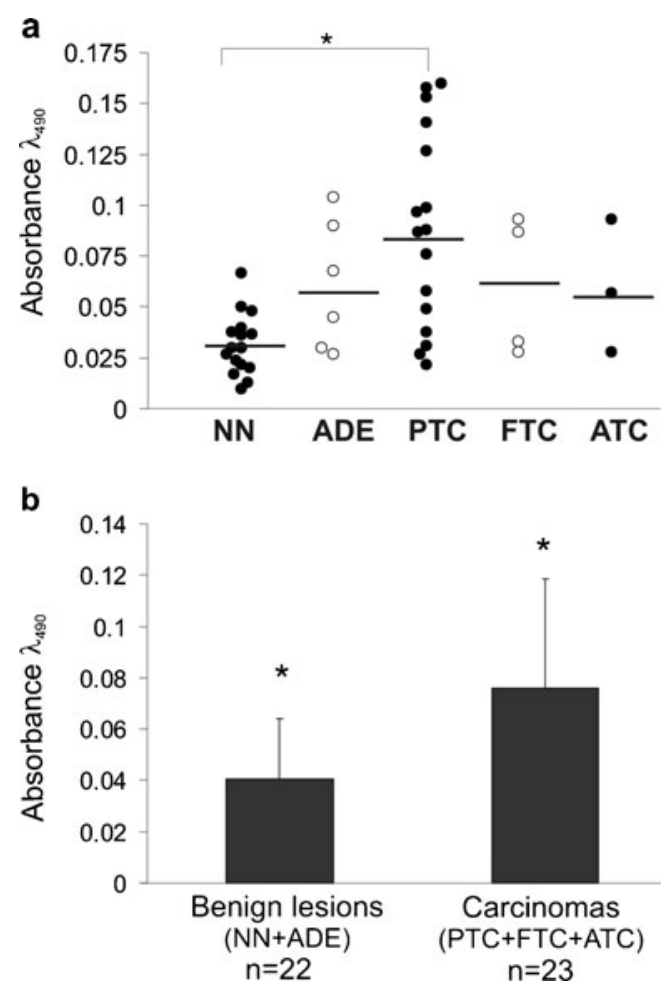

Fig. 1 Analysis of cytoplasmic Akt1 expression in thyroid pathological specimens by ELISA method. a Akt1 expression in individual cases of non-neoplastic lesions $(N N)$, adenoma $(A D E)$, follicular carcinoma $(F T C)$, papillary carcinoma $(P T C)$, and anaplastic carcinoma $(A T C)$; b Differences in Akt1 expression between benign and malignant lesions, error bars represents the mean $\pm \mathrm{SD}, p<0.05$

There were also differences in expression of Akt1 when benign and malignant lesions groups were compared (Fig. 1b). There were no statistically significant differences between adenomas and non-neoplastic lesions and between different types of cancers $(p>0.05)$. In most cases of thyroid pathological specimens, the expression of nuclear Aktl was very low and we were not able to detect it by ELISA method (data not shown).

Representative polypeptide patterns of nuclear and cytoplasmic proteins from thyroid lesions are shown in Fig. 2a, and the results of Akt1 identification among proteins of both fractions are shown in Fig. 2b. Akt1 both in cancer and benign lesions was localized mainly in cytoplasmic fraction. There were no significant differences in localization between differentiated cancers (papillary and follicular carcinomas) and benign lesions (non-neoplastic lesions and adenoma). In both types of lesions Akt1 was absent in nucleus or its expression was very low. However in two of three anaplastic cancer samples Akt1 was predominantly localized in nucleus and its expression was five to ten times higher than in the other samples (Fig. 2b).

Kinase Akt is well-known to be activated by phosphorylation at Ser473. We therefore examined the Akt1 

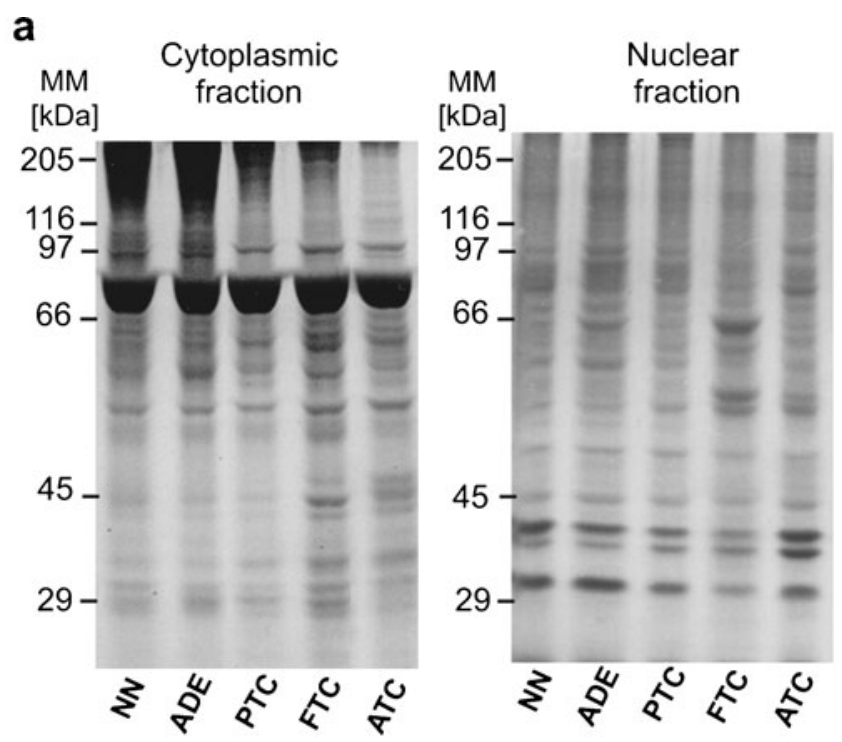

b

Cytoplasmic fraction

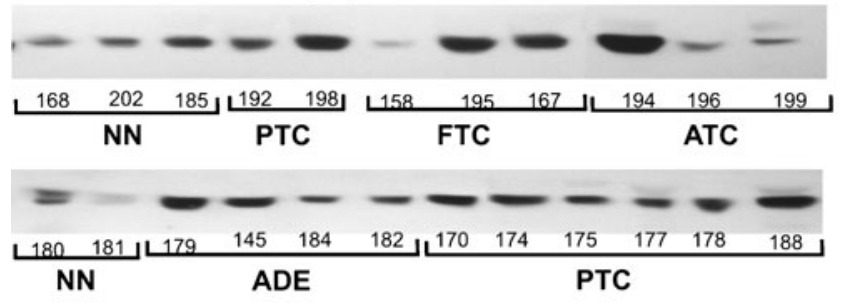

Nuclear fraction

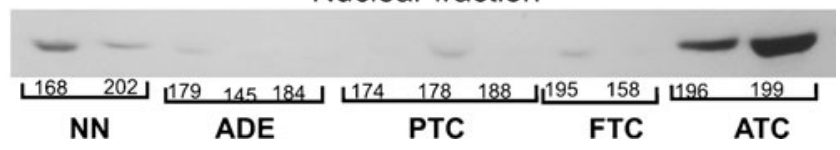

Fig. 2 Analysis of Akt1 expression in cytoplasmic and nuclear fractions from thyroid pathological specimens; a representative SDS polyacrylamide gel electrophoresis profiles of cytoplasmic and nuclear proteins; b Western blotting analysis of Akt1 in cytoplasmic and nuclear fractions, numbers indicate patient (samples) numbers

activation status in thyroid cancers and benign lesions by Western blot method using antibodies which recognize Akt phosphorylation at Ser473. However, the used antiphospho-Akt antibodies were non-isoform specific and recognized not only endogenous level of Akt1 isoform when phosphorylated at Ser473 but also recognized Akt2 and Akt3 when they are phosphorylated at the corresponding residues. Thus, Akt1 was first immunoprecipitated from cell lysates with monoclonal antiAkt1 antibody and then tested with anti-phospho Akt antibodies (Fig. 3a). The results in most thyroid cancer samples did not show higher phosphorylation of Akt1 than in benign lesions. On the contrary, the ratio of phosphorylated Akt1 to total Akt1 was usually lower in cancers than in non-neoplastic lesions and adenomas (Fig. 3b)

\section{Discussion}

We have analyzed here the expression, phosphorylation, and cellular localization of Akt1 in human benign and malignant thyroid lesions, i.e., nodular goiters, adenomas, papillary carcinomas, follicular carcinomas, and anaplastic carcinomas.

Kinase Akt is at the crossroads of a number of intracellular pathways and is a key signaling intermediate in growth and survival [4]. Overexpression and overactivation of Akt protein have been detected in aggressive human gastric cancers as well as breast, prostate, ovarian, and brain tumors [12]. Increased Akt protein expression and activity in a subset of human thyroid cancers in comparison to normal tissue have been also reported earlier. Ringel et al. [10] demonstrated that thyroid cancers (especially FTC) are characterized by increase expression of Akt1 and Akt2 and have increased levels of total Akt activity in comparison with normal tissue specimens. Akt has been frequently overexpressed and overactivated also in papillary carcinoma [13, 14].

Our results showing increased expression of Akt1 in the most cases of papillary, follicular, and anaplastic thyroid cancers in comparison with benign lesions are in agreement

\section{a}

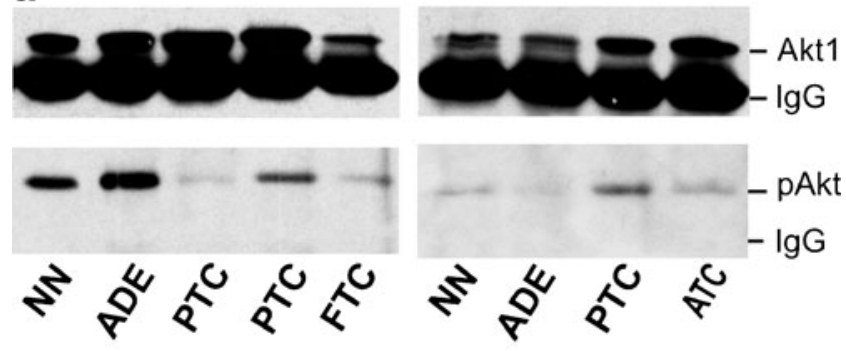

b

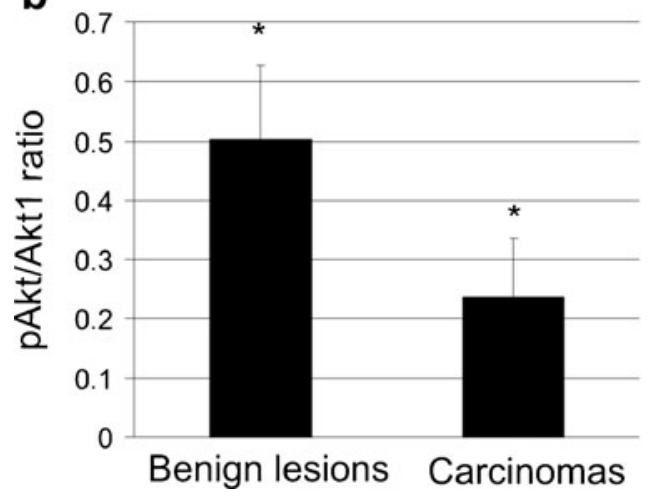

Fig. 3 Analysis of Akt1 phosphorylation. a Homogenate proteins of different thyroid pathological specimens were immunoprecipiteted with anti-Akt1 antibody and immunoprecipitates were immunobloted with anti-pAkt (Ser473) antibody or anti-Akt1 antibody. b Intensity of protein bands was quantified by densitometry and ratio of phosphoAkt1 to total Akt1 was estimated. Error bars represents the mean \pm SD 
with most previous data suggesting overexpression of Akt in cancers. Vasco et al. [11] showed that Akt activation and localization correlate with tumor invasion and oncogene expression in thyroid cancer. Immunoactive pAkt was greatest in regions of capsular invasion and was localized to the nucleus in follicular cancers and the cytoplasm in papillary cancers, except for invasive regions of papillary cancers where it was localized in both compartments. Immunoactive Akt1, but not Akt2 or Akt3, correlated with pAkt localization [11]. The results of our studies showed that Akt1 had mainly cytoplasmic localization in most benign and malignant thyroid pathological specimens. However, in two of three cases of anaplastic carcinoma, Akt1 had predominantly nuclear localization. These results could suggest that nuclear localization is characteristic only of very aggressive cancers. All cases of follicular carcinoma used in these studies showed the presence of capsular and vascular invasions, but there were no widely invasive cases. The papillary carcinoma cases were classified as stages I and II $\left(\mathrm{T}_{1-2} \mathrm{~N}_{0} \mathrm{M}_{0}\right)$ according to TNM staging system.

Our studies revealed the unexpected finding that thyroid cancers did not have higher phosphorylation of Ser473 of Akt1 than benign lesions. Moreover, the ratio of phosphoAkt1 to total Akt1 was even lower in cancer group than in adenomas and goiters. We do not know the reason of these discrepancies. But in earlier, mainly immunohistochemical studies usually, there was no differentiation made between Akt isoforms and total Akt phosphorylation. Thus, the activation of other Akt isoforms could increase total Akt activation. Moreover, Akt phosphorylation in cancers was compared with normal tissue and not with goiters or adenomas.

Recent studies have provided evidence for distinct functions of mammalian Akt isoforms in cancers, particularly with respect to the regulation of cell motility and metastasis.

Yoeli-Lerner et al. [15] revealed that expression of activated Akt1 potently blocks the in vitro migration and invasion of three distinct breast cancer cell lines through Matrigel. This effect required the kinase activity of Akt1 because an inactive catalytic site mutant did not block invasion. Similarly, Irie et al. [16] showed that silencing Akt1 expression enhanced cancer breast cell migration induced by IGF-1 and EGF and facilitated epithelial to mesenchymal transition. Hutchison et al. [17] reported that whereas expression of activated Akt1 in ErbB2 transgenic mouse mammary tumors enhanced their proliferation, it also resulted in fewer metastatic lesions compared with control animals. General Akt activation promotes metastasis in a mouse model of follicular thyroid carcinoma [18]. NPA cells (human papillary thyroid cancer cell line) have been shown to have Akt1-dependent cell migration. However, the degree of isoform-specific effects varies between the different thyroid cancer cell lines [2]. It can be presumed that Akt1 plays a role in cell proliferation and survival, but high activity of this isoform is not preferable for some cancer cells progression. Further studies are necessary to obtained more information regarding celltype dependent isoform expression and specificity in tumor progression to metastasis.

Acknowledgments This research was supported by grant No.301463534 from the Ministry of Science and Higher Education of Poland.

Open Access This article is distributed under the terms of the Creative Commons Attribution Noncommercial License which permits any noncommercial use, distribution, and reproduction in any medium, provided the original author(s) and source are credited.

\section{References}

1. Hou P, Liu D, Shan Y, Hu S, Studeman K, Condouris S, Wang Y, Trink A, El-Naggar AK, Tallini G, Vasko V, Xing M. Genetic alterations and their relationship in the phosphatidylinositol 3kinase/Akt pathway in thyroid cancer. Clin Cancer Res 13: 11611170, 2007.

2. Shinohara M, Chung YJ, Saji M, Ringel MD. AKT in thyroid tumorigenesis and progression. Endocrinology 148: 942-947, 2007.

3. Xing M. Genetic alteration in the phosphatidylinositol-3 kinase/ Akt pathway in thyroid cancer. Thyroid 20: 697-706, 2010.

4. Manning BD, Cantley LC. AKT/PKB signaling: navigating downstream. Cell 129, 1261-1274, 2007.

5. Matheny RW, Adamo ML. Current perspectives on Akt Aktivation and Akt-ions. Exp Mol Biol 234: 1264-1270, 2009.

6. Noguchi M, Ropars V, Roumestand C, Suizu F. Proto-oncogene TCL1: more than just a coactivator for Akt. FASEB J 21: 2273 2284, 2007.

7. Easton MR, Cho H, Roovers K, Shineman DW, Mizrani M, Forman MS, Lee VM-Y, Shablocs M, de Jong R, Oltrsdorf T, Ludwig T, Efsrtiadis A, Birnbaum MJ. Role of Akt3/protein kinase B in attainment normal brain size. Mol Cell Biol 25: 18691878, 2005.

8. Cho H, Mu J, Kim JK, Thorvaldsen JL, Chu Q, Crenshow EB, Keastner KH, Bartolomei MS, Hulman GI, Birnbaum MJ. Insuline resistance and a diabetes mellitus like syndrom in mice lacking the protein kinase Akt2 (PKB beta). Science 292: 17281731, 2001.

9. Cho H, Thorvaldsen JL, Chu Q, Feng F, Birnbaum MJ. Akt1/ PKBalpha is required for normal growth but dispensable for maintenance of glucose homeostasis in mice. J Biol Chem 276: 38349-38352, 2001.

10. Ringel MD, Hayre N, Saito J, Sounier B, Schuppert E, Burch H, Bernet V, Burman KD, Kohn LD, Saji M. Overexpression and overactivation of Akt in thyroid carcinoma. Cancer Res 61: 61056111, 2001.

11. Vasko V, Saji M, Hardy E, Kruhlak M, Larin A, Savchenko V, Miyakawa M, Isozaki O, Murakami H, Tsushima T, Burman KD, De Micco C, Ringel MD. Akt activation and localization correlate with tumour invasion and oncogene expression in thyroid cancer. $\mathrm{J}$ Med Genet 41: 161-170, 2004.

12. Chin YR, Toker A. Function of Akt/PKB signaling to cell motility, invasion and tumor stroma in cancer. Cell Signal 21: 470-476, 2009. 
13. Miyakawa M, Tsushima T, Murakami H, Wakai K, Isozaki O, Takano K, Increased expression of phosphorylated p70S6 kinase and Akt in papillary thyroid cancer tissues. Endocr J 50: 77-83, 2003.

14. Sozopoulos E, Litsiou H, Voutsinas G, Mitsiades N, Anagnostakis N, Tseva T, Patsouris E, Tseleni-Balafouta S. Mutational and immunohistochemical study of the PI3K/Akt pathway in papillary thyroid carcinoma in Greece. Endocr Pathol 21: 90-100, 2010.

15. Yoeli-Lerner M, Yiu GK, Rabinovitz I, Erhardt P, Jauliac S, Toker A. Akt blocks breast cancer cell motility and invasion trough the transcription factor NFAT. Mol Cell 20: 539-550, 2005.
16. Irie HY, Pearline RV, Grueneberg D, Hsia M, Ravichandran P, Kothari N, Natesan S, Brugge JS. Distinct roles of Akt1 and Akt2 in regulating cell migration and epithelial-mesenchymal transition. J Cell Biol 171: 1023-1034, 2005.

17. Hutchinson JN, Jin J, Cardiff RD, Woodgett JR, Muller WJ. Activation of Akt1 (PKB- $\alpha)$ can accelerated ErbB-2 mediated mammary tumorigenesis but suppresses tumor invasion. Cancer Res 64: 3171-3178, 2004.

18. Kim CS, Vasko VV, Kato Y, Kruhlak M, Saji M, Cheng SY, Ringel MD. AKT activation promotes metastasis in a mouse model of follicular thyroid carcinoma. Endocrinology 146: 4456-4463, 2005. 